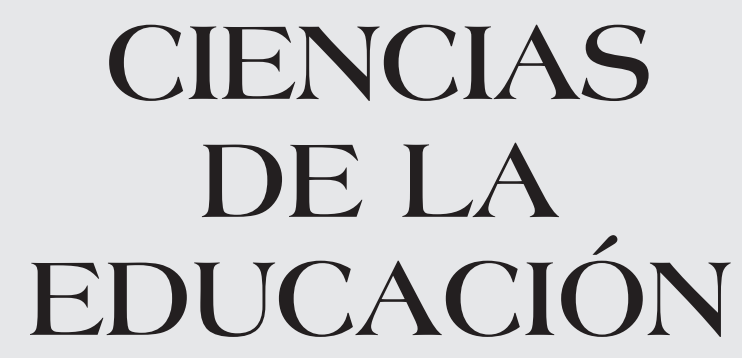





\title{
Efecto del uso de una estrategia pedagógica en el desarrollo de la conciencia moral en un grupo de estudiantes de educación media*
}

\section{EFFECT OF THE USE OF A PEDAGOGIC STRATEGY IN THE DEVELOPMENT OF THE MORAL CONSCIENCE IN A GROUP OF STUDENTS OF SECONDARY SCHOOL \\ EFFET DE L'UTILISATION D'UNE STRATÉGIE PÉDAGOGIQUE DANS LE DÉVELOPPEMENT DE LA CONSCIENCE MORALE CHEZ UN GROUPE D'ÉLÈVES DE LYCÉE}

\author{
Recibido: 15 de septiembre de 2010 • Aprobado: 15 de mayo de 2011 \\ María Sofía Bonilla Cárdenas ${ }^{* *}$ \\ Juan Ernesto Hernández Velandia*** \\ Magda Carolina Romero Contento**** \\ Claudia Fernanda Serrano Rodríguez $z^{* * * *}$ \\ Gabriel Alfonso Suárez Medina*****
}

Este trabajo es producto de la Línea de Investigación "Formación Integral” de la Maestría en Educación de la Universidad Santo Tomás. Fue clasificado como artículo de investigación.

** Licenciada en Educación Preescolar y Básica Primaria, Universidad Iberoamericana. Magíster en Educación, Universidad Santo Tomás. Contacto: sofia-bonilla@hotmail.com

*** Magíster en Educación, Universidad Santo Tomás. Contacto: juaner75@hotmail.com

**** Ingeniera Electrónica. Magíster en Educación, Universidad Santo Tomás. Contacto: carolinaromero05@gmail.com

***** Licenciada en Español e Inglés. Magíster en Educación, Universidad Santo Tomás. Contacto: clafe2008@hotmail.com

****** Magíster en Filosofía Latinoamericana, Universidad Santo Tomás. Doctor en Filosofía, Universidad Gregoriana de Roma. Docente investigador de la Maestría en Educación de la Universidad Santo Tomás. Contacto: gabrielsuarez@ustadistancia.edu.co 


\section{Resumen}

El presente artículo continúa con una línea problémica abierta en investigaciones anteriores aplicadas en Colombia sobre formación de la conciencia moral en la educación superior. Pero en esta oportunidad se enfoca un nuevo nivel: Ios estudiantes de educación media de la Institución Educativa Colegio Gilberto Alzate Avendaño de la ciudad de Villavicencio, en donde se implementa en el aula una estrategia pedagógica basada en dilemas morales, lo que potencialmente facilita el desarrollo de los niveles de conciencia moral. Para ello, se toma como referencia la escala de desarrollo socio-moral postulada por Lawrence Kohlberg, a fin de afianzar y enriquecer la capacidad de hacer juicios morales, en situaciones específicas, por parte de los adolescentes que participaron en esta investigación.

\section{Palabras clave}

Dilemas morales, conciencia moral, desarrollo moral, educación media, juicio moral.

\section{Abstract}

This article continues the problematical line opened in previous inquiries applied in Colombia about moral development in higher education. In this opportunity however the article approaches a new level, focusing on the students of the Gilberto Alzate Avendaño Secondary School in Villavicencio city, where a pedagogic strategy based in moral dilemmas which potentially facilitates the development of the levels of moral conscience is implementated in the classroom. To this end, the scale of socio-moral development by Lawrence Kohlberg is used as a reference in order to strenghten and enrich the ability to make moral judgments in specific situations of the teenagers who partook in this research.

\section{Keywords}

Moral dilemmas, moral conscience, moral development, secondary education, moral judgment.

\section{Résumé}

Cet article poursuit une ligne problématique ouverte dans des recherches préalables, appliquées en Colombie, sur la formation de la conscience morale dans l'enseignement secondaire et il aborde un nouveau niveau, se centrant, en l'occurrence, sur les lycéens de l'établissement "Colegio Gilberto Álzate Avendaño" de la ville de Villavicencio, au moyen de la mise en pratique dans la salle de classe d'une stratégie pédagogique axée sur les dilemmes moraux facilitant potentiellement le développement des niveaux de conscience morale. Pour atteindre ce but, l'échelle de développement socio-moral postulée par Lawrence Kohlberg a éte prise comme référence, visant à renforcer et à enrichir la capacité de porter des jugements moraux à des situations particulières, des adolescents ayant participé dans cette recherche.

\section{Mots clés}

Dilemmes moraux, conscience morale, développement moral, enseignement secondaire, jugement moral. 


\section{Introducción}

La línea problemática sobre desarrollo de la conciencia moral precisa de ejercicios permanentes y constantes de validación de la estrategia pedagógica basada en dilemas morales. Así, surge la pretensión de poner en práctica la propuesta generada por los investigadores Suárez y Meza (2005), lo que implica su ajuste, cualificación, corrección, afinación y puesta al servicio de la comunidad educativa.

Se presenta una estrategia concreta que parte de la relación entre teoría y práctica, para contribuir al enriquecimiento del quehacer formativo. Éste confronta una realidad de acuerdo con las formas de sentir, pensar y obrar de los educandos especialmente, quienes son los forjadores de una nueva generación. Por consiguiente, se quiere ofrecer una herramienta de trabajo aplicable, para contribuir con el cambio necesario que hay que instaurar en la educación en la línea de formación moral. Ésta no se puede comprender como una teoría simple del educar, sino como el saber cultivar y estimular los valores a través de las propias vivencias.

La pregunta que orientó la investigación se constituye en el hilo conductor de la misma y se encuentra expresada en los siguientes términos: "¿cuál es el efecto del uso de una estrategia pedagógica basada en la aplicación de dilemas morales en el desarrollo de la conciencia moral en estudiantes de educación media de la Institución Educativa Colegio Gilberto Alzate Avendaño de la ciudad de Villavicencio, departamento del Meta? En correspondencia con esta pregunta, el objetivo de la presente investigación es determinar el efecto de esa estrategia pedagógica en el desarrollo de la conciencia moral en los estudiantes de la institución señalada.

\section{Aproximación conceptual}

Los conceptos propios de esta investigación encuentran su mejor desarrollo, tanto en el marco teórico como en la propuesta pedagógica implementada que se trae a colación en el presente artículo, exponiendo algunas ideas que dan cuenta de estos.

La palabra "conciencia" (del latín conscire, conscientia, equivalente al griego sin-eidos) significa un cierto saber junto con o un conocer a la vez. Dos sentidos principales se le han dado a la palabra: 1) capacidad que tiene el hombre no sólo de conocer objetos, ya sean externos o internos a él, sino de advertir que conoce; 2) facultad de conocer el valor moral de los estados interiores y de los actos que el hombre pone como propios. "Conciencia", en general, designa el primer sentido. Para significar el segundo se emplea la expresión "conciencia moral". De igual modo, se puede decir que la conciencia es la facultad que permite dar cuenta si una conducta moral es valiosa o no.

Otro concepto es el referido a la formación de la conciencia. Para esta investigación, la adquisición de conocimientos no es el único fin de la inteligencia, ya que su manifestación más importante es la conciencia moral, es decir, la recta razón que dicta lo que se debe hacer cuando en pleno goce de libertad se hace necesaria una elección. En sentido similar se propone que la conciencia moral no es innata: se forma.

Finalmente, dado que la propuesta de formación moral está basada en el uso del dilema, se debe dar el significado o concepción de éste:

\footnotetext{
El dilema moral es una narración breve, a modo de historia, en la que se plantea una situación posible en el ámbito de la realidad, pero conflictiva a nivel moral. Por una parte, se solicita de los sujetos una solución razonada del conflicto y, de otra parte, un análisis de la solución elegida por el agente protagonista de la historia. Por regla general, la situación se presenta como una elección disyuntiva: el agente protagonista se encuentra ante una situación decisiva frente a la cual sólo existen dos, y nada más que dos, opciones, siendo ambas soluciones igualmente factibles o defendibles. El individuo se encuentra ante una verdadera e inevitable situación conflictiva (Suárez y Meza, 2005: 123).
} 


\section{Enfoque metodológico}

Esta investigación tiene un enfoque cuantitativo de orientación empírico-analítica, dirigida "a los fenómenos observables que sean susceptibles de medición, análisis estadístico y control experimental [...], enfatizando la evidencia empírica y cuantificación" (Arnal, del Rincón y Hernández, 1992: 84). Además, se desarrolló bajo el diseño cuasi-experimental. Al respecto, Hernández et ál. afirman:

La característica fundamental de este tipo de diseños está en que el investigador no puede hacer la asignación al azar de los sujetos a los grupos experimental y de control. Sin embargo, puede controlar alguna de las siguientes cuestiones: cuándo llevar a cabo las observaciones, cuándo aplicar la variable independiente o tratamiento y cuál de los grupos recibirá el tratamiento. Aunque estos diseños no garantizan un nivel de validez interna y externa como en los experimentales, ofrecen un grado de validez suficiente, lo que hace muy viable su uso en el campo de la educación y de la psicopedagogía (1998: 101).

Dentro de los diseños cuasi-experimentales posibles se asumió el correspondiente a grupos de control no equivalentes, con pretest y postest.

Determinado el método y la estrategia a implementar, se procedió a considerar los diferentes instrumentos para evaluar el desarrollo moral de un sujeto. A partir del análisis de estos instrumentos, el grupo pasó a la utilización del cuestionario de problemas socio-morales de James Rest (1986), el más popular de ellos, que, además, está basado en la teoría evolutiva de Lawrence Kohlberg ${ }^{1}$.

\section{Resultados y discusión}

A continuación se mostrará el análisis comparativo pretest-postest, tanto del grupo experimental como del grupo de control.

\section{Análisis comparativo pretest-postest del grupo experimental}

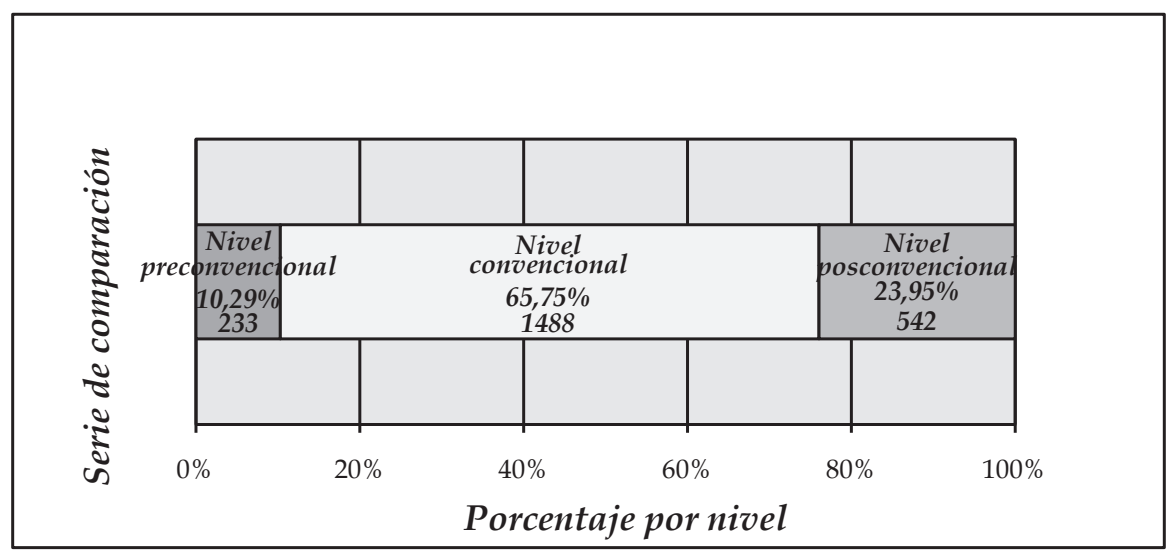

Gráfica 1. Comparación pretest por niveles del grupo experimental

$1 \quad$ El modelo de desarrollo propuesto por Kohlberg (1984) para describir el razonamiento moral ha sido casi hegemónico en el área de la psicología cognitiva ocupada del estudio del juicio y las justificaciones sobre asuntos socio-morales. Una de las razones para ocupar esta posición hegemónica es el importante trabajo realizado con el fin de contar con instrumentos de recolección de datos, que permitan comprobar las bondades de la propuesta teórica (Suárez y Meza, 2005). 


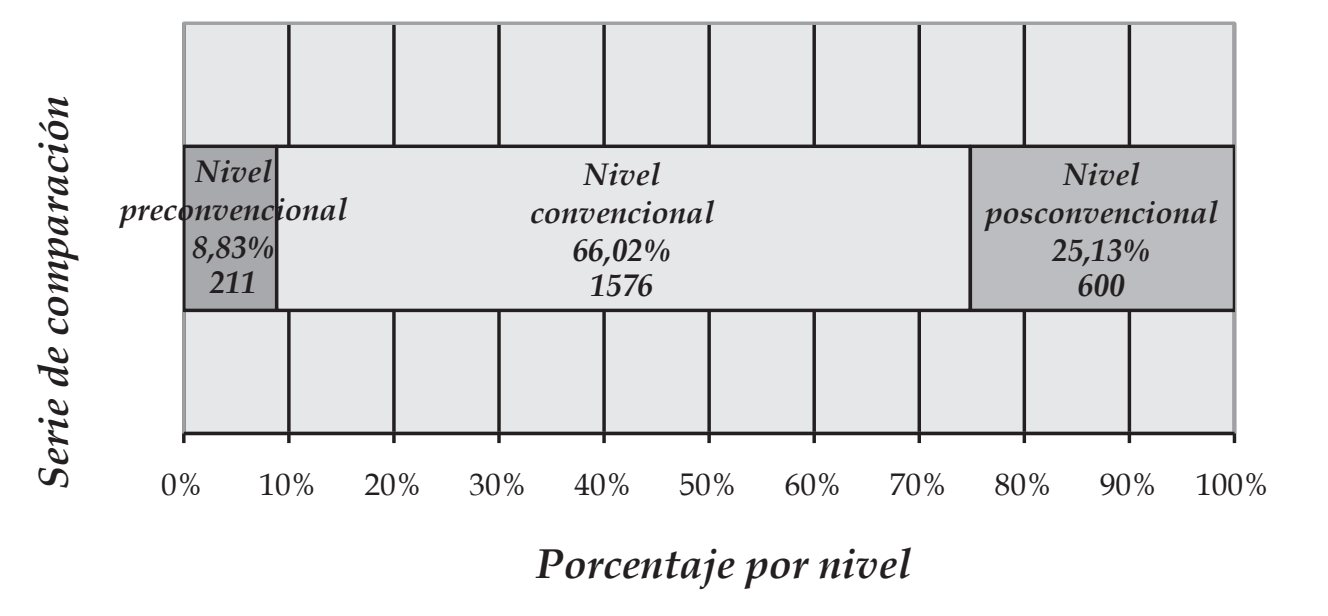

Gráfica 2. Comparación postest por niveles del grupo experimental

El punto de referencia de la efectividad de la estrategia pedagógica es el grupo experimental, que para el momento del pretest no había tenido ningún contacto con el grupo de trabajo o con metodologías similares. En general, el puntaje del pretest en el nivel preconvencional fue de 233 puntos, cuya representación porcentual es de 10,29\%. En este conjunto de valoraciones, el grupo obtuvo un puntaje de 1488 puntos en el nivel convencional, correspondientes al 65,75\%. Por último, en el nivel posconvencional se obtuvieron 542 puntos, equivalentes a un $23,95 \%$.

Al observar los puntajes obtenidos en los distintos niveles se infiere que el grupo se ubicó en esta primera etapa en el nivel convencional, caracterizado por seguir la perspectiva de un individuo perteneciente a un grupo, institución, sociedad; por actuar de acuerdo con las normas morales que rigen en la comunidad a la que pertenecen; por esforzarse por cumplir bien con el papel que desempeña e, igualmente, por salvaguardar los intereses sociales y propios (Hersh, Reimer y Paolitto, 2002: 57).

Luego de la intervención, los co-investigadores aplicaron el postest, del que se pudo concluir, basándose en las gráficas 1 y 2, que el grupo en el nivel preconvencional disminuyó de 233 a 211 puntos; en cuanto al nivel convencional, los puntajes aumentaron de 1488 a 1576, y en el nivel posconvencional la puntuación aumentó de 542 a 600 puntos. Con ello se demuestra que luego de la aplicación de la estrategia pedagógica, el grupo experimental, aunque continúa ubicándose en el nivel convencional, evidenció una evolución en el desarrollo de su conciencia moral, asumiendo convenientemente la metodología sugerida con base en el uso de dilemas morales, mejorando su capacidad de argumentación de juicios morales, presentando una apertura hacia la discusión de temas sensibles y progresando en la capacidad de escucha de los compañeros.

En consecuencia, las gráficas indican que los estudiantes se sirvieron del proceso de toma de perspectiva para aumentar su comprensión y capacidad de reflexión frente a situaciones sociomorales sensibles, tal como lo indica la teoría de Kohlberg. Además, aunque ningún estudiante del grupo se ubicó en el nivel posconvencional, sí se evidenció el aumento del puntaje en dicho nivel, indicando una tendencia hacia el progreso o evolución. 
Los resultados del análisis gráfico también pueden ser corroborados con las argumentaciones dadas por cada uno de los estudiantes a las situaciones socio-morales presentadas, las cuales están consignadas en el formato de Registro de Respuestas Individuales como evidencia para demostrar la evolución en su capacidad de generar juicios morales. Así, se identificaron 3 grupos:

a) Estudiantes que mostraron evolución en el desarrollo del juicio moral. Dentro de este grupo se encuentran ubicados específicamente 8 estudiantes, equivalente al $30,76 \%$ de la muestra, que corresponden a los códigos 31, 36, 37, 40, 46, 48, 55 y 61 . Estos estudiantes evidenciaron progresivamente, en primer lugar, una argumentación asentada en el respeto por las normas, basada en la aspiración de lograr una sociedad mejor a través de este mecanismo.

Por ejemplo, el código 48 afirma en su respuesta al dilema 22: "Yo de ella le pediría el apartamento, ya que este señor presenta problemas hacia la comunidad; no sé, de pronto llegue a presentarse algo. Pero lo mejor es que desocupe, que se vaya, para la seguridad del sector"3. En esta respuesta se observa que el estudiante prioriza el bienestar de la comunidad sobre cualquier interés particular, lo que demuestra que toma una perspectiva social que privilegia el punto de vista de la sociedad, así como los acuerdos o motivos interpersonales, porque considera el punto de vista de un sistema que tiene un orden y una organización establecida.

De igual manera, en el dilema $6^{4}$ una estudiante responde: "Pues de mi parte, no me gustaría esa clase de trabajo, porque la idea de un aborto está mal, ya que se trata de la vida de un niño"5; quien después de la discusión grupal continúa con su

2 Cfr. Informe Final del Proyecto de Investigación, anexo 2, dilema 2: El alquiler.

3 Cfr. Informe Final del Proyecto de Investigación, anexo 4, código 48, dilema 2.

4 Cfr. Informe Final del Proyecto de Investigación, anexo 2, dilema 6: Laura.

5 Cfr. Informe Final del Proyecto de Investigación, anexo 4, código 48, dilema 6 . posición, afirmando: "Sí, yo mantengo mi decisión, ya que es una vida la que estamos destruyendo, y está mal ese trabajo, no me gustaría"6. En el trasfondo de esta última afirmación se evidencia un respeto al derecho a la vida, considerado como derecho universal.

Por otro lado, estos estudiantes manifiestan un respeto hacia las autoridades consolidadas, debido a que delegan en ellas la solución a los problemas que se les presentan, sobre todo en los dilemas relacionados con normas de tránsito, procesos penales y bioética. Esto se puede observar en las afirmaciones realizadas por el estudiante de código 31, quien manifiesta como respuesta al dilema 17: "Tomás tiene sentimientos de culpa por la señora, pero a Mario no se le dio nada. Yo pienso que los dos son culpables del accidente y deberían ir a la Policía y aclarar todo lo que pasó; sobre todo, pedirle perdón a la señora por la imprudencia de manejar en estado de embriaguez, y no volverlo a hacer, aprender de sus errores y corregirlos" $"$.

Además, a lo largo del desarrollo de sus argumentaciones en torno a los dilemas planteados mostraron un interés por cumplir los deberes asignados y las normas vigentes -exceptuando aquellas situaciones que entraran en contravía con la legitimidad de la existencia y del derecho a la vida de las personas-, así como por mostrar un imperativo de conciencia colectiva. La creencia en las normas obedece, ante todo, a un marcado interés por mantener el funcionamiento de un sistema social y de valores dado, que para estas personas marca el rumbo de sus juicios morales e influye en su capacidad para solucionar problemas socio-morales cotidianos.

6 Cfr. Informe Final del Proyecto de Investigación, anexo 4, código 48, dilema 6 .

7 Cfr. Informe Final del Proyecto de Investigación, anexo 2, dilema 1: Tomás fue testigo.

8 Cfr. Informe Final del Proyecto de Investigación, anexo 4, código 31, dilema 1. 
Lo anterior se ve reflejado en la respuesta de una estudiante en la sesión 49, que afirma: "Si yo supiese que en esa empresa explotan a los niños, no compraría ropa allá, por muy barata que estuviera, porque es injusto. La gente que sabe eso no debería ser cómplice de esos maltratos y explotaciones y debería denunciar"10. La respuesta denota un marcado interés por el consumo responsable en empresas, que no afecte al colectivo ni directa ni indirectamente.

b) Estudiantes que mantuvieron su desarrollo del juicio moral. En este apartado se hace alusión a los estudiantes que por los resultados, tanto del pretest como del postest, y por su razonamiento moral, expuesto en las diferentes argumentaciones dadas durante el proceso de intervención de la propuesta pedagógica, fueron ubicados específicamente en el estadio 3 ó 4 de este nivel y que, al mismo tiempo, se mantuvieron en estos. Así, se identificó que 9 estudiantes (34,61\% de la muestra), correspondientes a los códigos $29,30,32,43,44,45,49,58$ y 59 , se ubicaron en el estadio 3 ; y que 8 estudiantes $(30,76 \%$ de la muestra), correspondientes a los códigos 33 , $38,39,41,47,51,57$ y 62 , se mantuvieron en el estadio 4 de comienzo a fin del proceso.

Dentro de los estudiantes que se mantuvieron en el estadio 3 se puede tomar como ejemplo el caso del código 59, quien responde al dilema 111: "Mario debería tomar esta situación con responsabilidad. Si éste no lo hace, pensamos que estaría bien que Tomás lo denunciara a las autoridades"12. Se evidencia aquí la tendencia al mantenimiento de las reglas y el reconocimiento de una autoridad como apoyo a la típica buena conducta. Esta característica del estadio 3 igualmente se evidencia en la respuesta dada al dilema

9 Cfr. Informe Final del Proyecto de Investigación, anexo 2, dilema 4: Trabajo infantil.

10 Cfr. Informe Final del Proyecto de Investigación, anexo 4, código 31, dilema 4.

11 Cfr. Informe Final del Proyecto de Investigación, anexo 2, dilema 1: Tomás fue testigo.

12 Cfr. Informe Final del Proyecto de Investigación, anexo 4, código 59, dilema 1.
313: "Definitivamente debe indultarlo, porque ya cumplió su condena"14, en la que se identifica la obediencia a las leyes establecidas y el respeto y cumplimiento a lo estipulado por las autoridades.

En cuanto a la respuesta dada al final del proceso, que permite evidenciar que este estudiante permaneció en el estadio 3, se puede tomar como ejemplo su argumentación respecto al dilema 6"15: "Hay que hacer cualquier cosa para subsistir y más cuando se trata de uno mismo. Muy personalmente creo que ella debería aceptar la invitación al trabajo de la doctora. Cuando se trata de rebuscar lo que sea para poder vivir no vale la religión ni el miedo, aunque hay que tener en cuenta que el trabajo en el que uno esté no haga daño a las demás personas"16. Se puede resaltar que el estudiante asume el lugar del otro para entender la situación y que hay un interés preponderante por la vida, pero también por lo que está bien para los demás.

Además, las respuestas de estos estudiantes se caracterizan, de manera general, por un respeto irrestricto hacia los cánones y normas establecidas por la sociedad. Sus argumentaciones se encuentran amparadas en principios ético-religiosos que hacen parte de su entorno particular. De igual manera, en el trabajo en grupo se detectó una tendencia a tomar cambios de perspectiva después de adoptar una decisión en torno a la solución de un dilema propuesto, sobre todo en aquellos casos relacionados con los temas de bioética y trabajo infantil. Así mismo, se muestra firmeza en cuanto a las posiciones tomadas en asuntos penales y judiciales, como los dilemas 1, 2, 3 y 5. Por otro lado, en sus respuestas a los dilemas planteados en las intervenciones se manifiesta una conciencia de su individualidad y la de sus

13 Cfr. Informe Final del Proyecto de Investigación, anexo 2, dilema 3: Luis.

14 Cfr. Informe Final del Proyecto de Investigación, anexo 4, código 59, dilema 3.

15 Cfr. Informe Final del Proyecto de Investigación, anexo 2, dilema 6: Laura.

16 Cfr. Informe Final del Proyecto de Investigación, anexo 4, código 59, dilema 6. 
semejantes, mostrando buenos motivos e interés por los demás; características propias del estadio 3 del nivel convencional.

Respecto a los estudiantes que se mantuvieron en el estadio 4, se evidenció la acogida de "la perspectiva del sistema social en el que participan", que es una característica del estado 4 del nivel convencional (Hersh, Reimer y Paolitto, 2002: 65). En este caso, es la perspectiva de nuestra sociedad con respecto a las normas de tránsito, que son ponderadas por los argumentos dados en el primer dilema, manifestando la pertinencia de no manejar embriagado y atenerse a las consecuencias de hacerlo; en el dilema $2^{17}$ el sistema de creencias fue ponderado por los estudiantes mediante la concepción religiosa de "pecado" y la argumentación dada en procura de una solución ${ }^{18}$.

Igualmente, otra característica que evidencia la ubicación y permanencia de estos estudiantes en el estadio 4 se confirma con el dilema $4^{19}$, en el que se señala ir en contra de la ilegalidad, "porque no es bueno contribuir con la explotación infantil; esto es un mal para la sociedad"20; es decir, de acuerdo con Kohlberg (1985), detener esta situación es ser bueno, como también ser bueno es procurar el mantenimiento de la buena marcha de la sociedad, independientemente de los propios intereses y de cómo se es visto por los otros (Hersh, Reimer y Paolitto, 2002: 56). Para ello se debe tener en cuenta los sentimientos y expectativas de los individuos implicados, que en este caso son los niños trabajadores.

c) Estudiantes que descendieron en el desarrollo del juicio moral. En este grupo se ubica sólo 1 estudiante de los 26 que conforman la muestra (3,84\%), correspondiente al código 52. Aun cuando

17 Cfr. Informe Final del Proyecto de Investigación, anexo 2, dilema 2: El alquiler.

18 Cfr. Informe Final del Proyecto de Investigación, anexo 4, dilema 6, código 57.

19 Cfr. Informe Final del Proyecto de Investigación, anexo 2, dilema 4: Trabajo infantil.

20 Cfr. Informe Final del Proyecto de Investigación, anexo 4, código 59, dilema 4. permaneció en el nivel convencional tanto en el pretest como en el postest, al aplicar este último se mostró una disminución en los puntajes, lo que representa una reubicación del estadio 4 al estadio 3. En este caso, el proceso de intervención identificó una marcada tendencia a tomar como propio el juicio moral de un grupo o colectividad, lo que se puede visualizar mediante su respuesta inicial al dilema 621: "Yo creo que debería vender su embrión, ya que ella y su familia están en escasez económica"22. Pero su afirmación, una vez realizada la sesión grupal, cambia por un "no, porque hay varios medios de sobrevivir no matando vidas"23.

De igual manera, es proclive a demostrar un nivel de argumentación con poca profundidad, lo que se evidencia en su respuesta al dilema 524: "La segunda, la autorizacion pensando en la audiencia" 25 o en la respuesta dada al dilema 126: "Tomás debería convencer a su amigo de ir a las autoridades y afrontar el problema como se debe"27.

Además, este estudiante presentó una confusión en la solución de los problemas planteados en los dilemas, lo que se refleja en su respuesta al dilema 428: "Sí, compraría la ropa y llevaría ante las autoridades la queja de la explotacion de los niños"29. Incluso después de la sesión grupal

21 Cfr. Informe Final del Proyecto de Investigación, anexo 2, dilema 6: Laura.

22 Cfr. Informe Final del Proyecto de Investigación, anexo 4, código 52, dilema 6.

23 Cfr. Informe Final del Proyecto de Investigación, anexo 4, código 52, dilema 6.

24 Cfr. Informe Final del Proyecto de Investigación, anexo 2, dilema 5: Prensa rosa.

25 Cfr. Informe Final del Proyecto de Investigación, anexo 4, código 52, dilema 5.

26 Cfr. Informe Final del Proyecto de Investigación, anexo 2, dilema 1: Tomás fue testigo.

27 Cfr. Informe Final del Proyecto de Investigación, anexo 4, código 5, dilema 1.

28 Cfr. Informe Final del Proyecto de Investigación, anexo 2, dilema 4: Trabajo infantil.

29 Cfr. Informe Final del Proyecto de Investigación, anexo 4, código 52, dilema 4. 
afirmó: "Sí, porque si los niños estan trabajando es por una necesidad economica; no necesariamente tienen que estar obligados" 30 . Lo anterior no sólo muestra una confusión en las respuestas, sino una dispersión de las ideas apropiadas para la solución de un dilema. También los coinvestigadores detectaron durante el proceso de intervención y aplicación de los instrumentos una actitud despreocupada y desinteresada en cada una de las sesiones; inclusive en algunas ocasiones hubo una tendencia a interrumpir al grupo.

\section{Análisis comparativo pretest-postest del grupo de control}

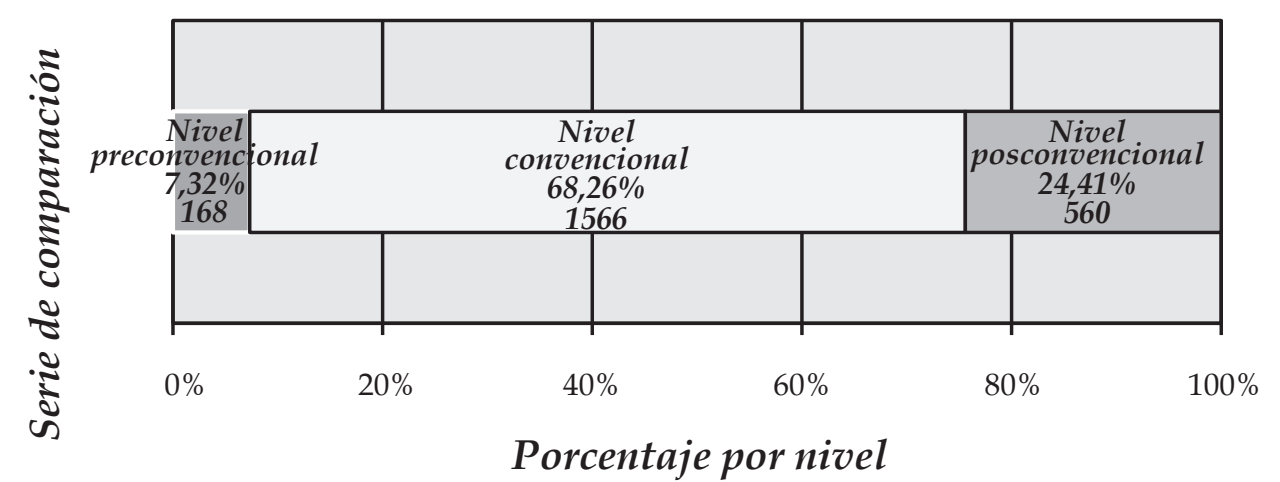

Gráfica 3. Comparación pretest por niveles del grupo de control

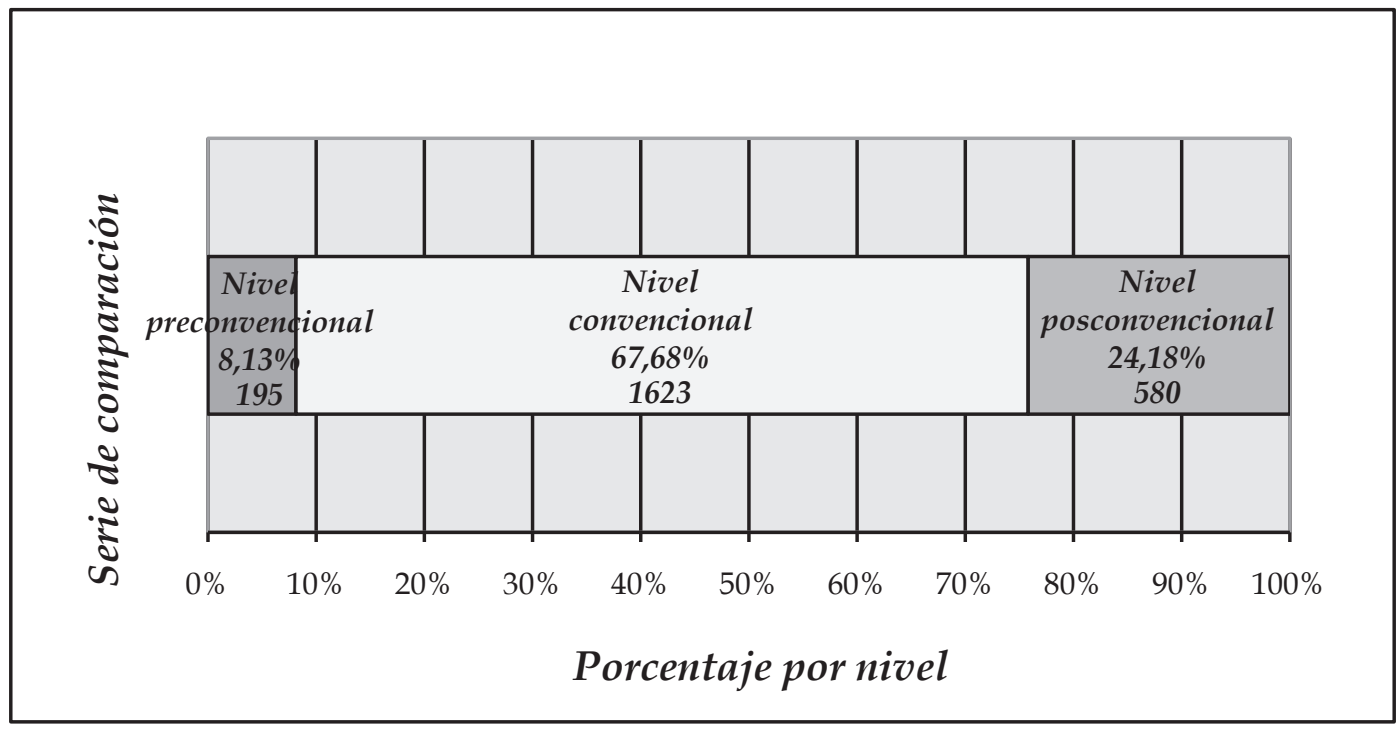

Gráfica 4. Comparación postest por niveles del grupo de control

30 Cfr. Informe Final del Proyecto de Investigación, anexo 4, código 52, dilema 4. 
En la primera etapa de aplicación del pretest se estableció el nivel de desarrollo de la conciencia moral de los estudiantes del grupo de control, en el que se obtuvieron los siguientes puntajes: en el nivel preconvencional, 168 puntos, equivalentes al 7,32\%; en el nivel convencional, 1566 puntos, equivalentes a $68,26 \%$, porcentaje que indica una mayor tendencia del grupo a ubicarse en este nivel; en el nivel posconvencional, 560 puntos, correspondientes al $24,41 \%$.

Después de la aplicación del postest al grupo de control, y habiendo transcurrido un año lectivo desde la aplicación del pretest, se evidenció, como se muestra en las gráficas 3 y 4 , que el grupo que no fue sometido a la intervención con la estrategia pedagógica propuesta continuó en el mismo nivel convencional, aunque aumentó su puntaje en cada uno de los niveles. El aumento, en comparación con el pretest, fue de 27 puntos en el nivel preconvencional, de 57 puntos en el nivel convencional y de 20 puntos en el nivel posconvencional.

\section{Análisis comparativo entre el grupo de control y el grupo experimental}

Atendiendo la necesidad de realizar la comparación del nivel de desarrollo de la conciencia moral entre el grupo de estudiantes que recibió la estrategia pedagógica basada en dilemas morales y el grupo que no la recibió, los co-investigadores identificaron las siguientes variaciones en los puntajes obtenidos en cada uno de los niveles:

Nivel preconvencional: mientras que en el grupo experimental el puntaje disminuyó en 22 puntos, en el grupo de control aumentó en 27 puntos, lo que permite evidenciar que el grupo que recibió la estratregia pedagógica tendió a aumentar su capacidad de desarrollo del juicio moral, al abandonar un nivel caracterizado por una moralidad heterónoma y un individualismo instrumental, el cual está claramente orientado hacia la no consideración de los intereses de sus semejantes y hacia el no el reconocimiento de la diferencia. De igual manera, el indivudualismo presente en este nivel reconoce el conflicto de intereses. Cabe anotar que los grupos, tanto en el pretest como en el postest, superaron este nivel, aunque se notó en el grupo experimental, gracias a la intervención realizada, la tendencia a alejarse de éste, en comparación con el grupo de control.

Nivel convencional: el grupo experimental aumentó su puntuación en 88 puntos en comparación con el grupo de control, cuyo incremento fue de 57 puntos. Esto corrobora que la estrategia aportó al desarrollo del juicio moral de los estudiantes un conjunto de herramientas de análisis de las diferentes situaciones socio-morales presentadas durante el proceso de intervención. En este sentido, es posible indicar que la tendencia más fuerte es alejarse del nivel preconvencional y ubicarse en el nivel convencional con mayor puntuación, lo que resalta una moralidad enmarcada por vivir de acuerdo con lo que las personas próximas o en general esperan de quienes ejercen determinados roles. "Ser bueno" es importante, significa tener buenos motivos, preocuparse por los demás, mantener relaciones mutuas tales como confianza, lealtad, respeto y gratitud. Adicionalmente, es buscar cumplir los deberes con los que se está de acuerdo y defender las leyes, salvo en casos extremos en que entren en conflicto con otros deberes sociales establecidos. En síntesis, es bueno contribuir a la sociedad, al grupo o a la institución.

Nivel posconvencional: el grupo experimental aumentó en 58 puntos en comparación con el grupo de control, que aumentó en 20 puntos. El aumento presentado por el grupo experimental obedeció a que una intervención basada en dilemas morales posibilitó a los estudiantes argumentaciones y reflexiones más fundamentadas y una evolución en el desarrollo del juicio moral, logrando la adquisición de una mayor conciencia acerca de la variedad de valores y opiniones enmarcadas en las reglas propias del grupo. 


\section{Conclusiones}

El desarrollo de la presente investigación surgió a partir de la propuesta generada por los investigadores Gabriel Suárez y José Luis Meza (2005), titulada "Estudio del desarrollo de la conciencia moral y propuesta para su formación en la educación superior". Dicha investigación se encuentra vinculada a la Línea de Investigación "Formación Integral" de la Maestría en Educación de la Universidad Santo Tomás.

Esta investigación se circunscribió en el nivel de educación Media Técnica y se aplicó en la Institución Educativa Colegio Gilberto Alzate Avendaño de la ciudad de Villavicencio. El hecho de implementarse en dos grupos de este nivel educativo vislumbra una intención de ampliar la aplicación de esta estrategia pedagógica.

La respuesta que da el grupo de co-investigadores a la pregunta inicial planteada en la investigación se orienta a concluir que la estrategia pedagógica implementada surte un efecto positivo en el desarrollo de la conciencia moral de los estudiantes que hacen parte del grupo experimental, lo que se demuestra mediante el incremento de los puntajes en el nivel convencional y posconvencional. De la misma manera, esta evolución en el desarrollo moral se ve expresada en las argumentaciones dadas por los estudiantes. Dicho efecto conlleva comprobar la hipótesis planteada: los estudiantes partícipes del uso de la estrategia pedagógica basada en la aplicación de dilemas morales mostraron un mayor desarrollo de la conciencia moral, en comparación con aquellos que no participaron de ésta.

De la misma manera, luego de la aplicación del instrumento de medición, de recopilar las argumentaciones de los estudiantes y de realizar un análisis exhaustivo a los resultados obtenidos, se da cumplimiento al objetivo general, tal como se puede evidenciar en el apartado "Análisis comparativo pretest-postest del grupo experimental".
Con respecto al segundo objetivo de la investigación-poner en práctica la estrategia pedagógica basada en dilemas morales en estudiantes de educación media-, se logró la puesta en práctica de la estrategia pedagógica a lo largo de seis sesiones organizadas en diferentes fechas del año.

A partir de los resultados obtenidos en el pretest y el postest, y dando cumplimiento al tercer objetivo, se realizó la comparación del nivel del desarrollo de la conciencia moral entre el grupo que recibió una estrategia pedagógica basada en dilemas morales y el grupo que no la recibió, lo que hizo posible confirmar la efectividad de la estrategia pedagógica implementada, tal y como se expresa de forma más específica en el apartado "Análisis comparativo entre el grupo de control y el grupo experimental".

En cuanto a los resultados alcanzados, se concluye que luego de analizar la variación de los puntajes obtenidos por el grupo experimental en el pretest y postest se detectó en los tres niveles una disminución de 22 puntos en el nivel preconvencional y un aumento de 88 y 58 puntos en el nivel convencional y posconvencional, respectivamente. Esto pone de manifiesto la evolución en la conciencia moral de los estudiantes intervenidos y corrobora, una vez más, la efectividad de la estrategia, tal y como se puede evidenciar en las gráficas 1 y 2.

Igualmente, en lo referente al análisis comparativo pretest-postest del grupo de control, se puede concluir que éste presentó una regresión en el puntaje preconvencional. Aunque también en este grupo aumentaron los puntajes del nivel convencional y posconvencional en 57 y 20 puntos respectivamente, los resultados no fueron tan significativos como los obtenidos por el grupo experimental (ver gráficas 3 y 4 ).

Pese a que ambos grupos se ubicaron en el nivel convencional, luego de la aplicación del postest se evidenció un incremento en los puntajes obtenidos por el grupo experimental, en comparación con 
el grupo de control. Además, la puntuación del grupo experimental en el nivel preconvencional decreció en comparación con el puntaje obtenido por el grupo de control, el cual se incrementó, tal y como se puede evidenciar en las gráficas 2 y 4. En el caso del puntaje posconvencional obtenido en el postest, se observó que en los dos grupos hubo incremento de los puntajes, pero específicamente en el grupo experimental este incremento fue mayor, convirtiéndose en un indicador más de la eficacia de la estrategia.

Así mismo, la disposición de la mayoría de los estudiantes del grupo intervenido, así como la apropiación y estructura de la estrategia, permitieron un trabajo organizado que corrobora el alcance e impacto de la propuesta pedagógica. Finalmente es pertinente señalar que una vez culminada la presente investigación se abre la posibilidad de enriquecer la estrategia pedagógica en el proceso de su aplicación, como también vincularla a currículos propios de la educación media, no solamente en el área de ética, sino también como proyecto transversal que contribuya a la formación integral.

\section{Referencias}

Arnal, del Rincón y Latorre (1992). Investigación educativa. Fundamentos y metodologías. Barcelona: Labor.

Buendía, L., Colás, M. y Hernández, F. (1998). Métodos de investigación en psicopedagogía. Madrid: McGraw-Hill.

Hers, R., Reimer, J. y Paolitto, D. (2002). El crecimiento moral de Piaget a Kohlberg. Madrid: Narcea.

Kohlberg, L. (1985). Estadios morales y moralización, la vía cognitivo-evolutiva. Psicología del desarrollo. Barcelona: Desclée de Brouwer.

Rest, J. (1986). DIT. Manual for the Definig Issues Test. Mineapolis, University of Minessota, Center for the Study of Ethical Development.

Suárez, G. y Meza, J. (2005). Conciencia moral. Estudio del desarrollo de la conciencia moraly propuesta para su formación en la educación superior. Bogotá: Universidad de La Salle. 